\title{
Nonexponentiality of time dependent survival probability and the fractional viscosity dependence of the rate in diffusion controlled reactions in a polymer chain
}

\author{
Goundla Srinivas \\ Solid State and Structural Chemistry Unit, Indian Institute of Science, Bangalore, India 560012 \\ Arun Yethiraj ${ }^{\mathrm{a})}$ \\ Department of Chemistry, University of Wisconsin, Madison, Wisconsin 53706 \\ Biman Bagchi ${ }^{\text {b) }}$ \\ Solid State and Structural Chemistry Unit, Indian Institute of Science, Bangalore, India 560012
}

\begin{abstract}
Brownian dynamics (BD) simulations have been carried out for the time dependent survival probability $\left[S_{p}(t)\right]$ of donor-acceptor pairs embedded at the two ends of an ideal polymer chain. Long distance fluorescence resonance energy transfer (FRET) between the donor and the acceptor is assumed to occur via the Forster mechanism, where the transfer rate $k(R)$ is a function of the distance $(R)$ between the donor and acceptor. For the Rouse chain simulated here, $k(R)$ is assumed to be given by $k=k_{F} /\left[1+\left(R / R_{F}\right)^{6}\right]$, where $k_{F}$ is the rate in the limit of zero separation and $R_{F}$ is the Forster radius. The survival probability displays a strong nonexponential decay for the short to intermediate times when $R_{F}$ is comparable to $R_{M}$ [distance at which the $R^{2} P(R)$ is maximum]. The nonexponentiality is also more prominent in the case of highly viscous polymer solutions. It is predicted that the FRET rate can exhibit a fractional viscosity dependence. This prediction can be tested against experiments. We have also compared the BD simulation results with the predictions of the well-known Wilemski-Fixman (WF) theory at the level of survival probability. It is found that the WF theory is satisfactory for the smaller $R_{F}$ values (where the rate is small). However, the agreement becomes progressively poorer as the Forster radius is increased. The latter happens even at intermediate strengths of $k_{F}$. The present results suggest the need to go beyond the WF theory.
\end{abstract}

\section{INTRODUCTION}

Fluorescence resonance energy transfer (FRET) is a powerful technique for the study of many aspects of structure and dynamics of polymers and biopolymers in solution. ${ }^{1-8}$ In this technique, the polymer is doped with a donor and an acceptor site at suitable positions along the chain backbone. The donor is excited optically by laser light and the energy transfer to the acceptor is monitored. When the distance between the donor-acceptor (DA) pair is fixed, as is the case in rigid biopolymers, ${ }^{6,7}$ the FRET experiment provides information on the distance between the donor and acceptor sites, since the mechanism for energy transfer is generally known. For flexible molecules in solution, the distance between the donor and acceptor sites is a fluctuating quantity and, therefore, FRET experiments can be used to obtain detailed information on the conformational dynamics of the individual molecules. For example, the folding dynamics of proteins can be studied directly using this technique. ${ }^{8}$ Clearly, the use of FRET in single molecule spectroscopy of polymers and biopolymers requires theoretical understanding of the dependence of the survival probability on the fluctuating distance between the DA pair. ${ }^{9-11}$ Actually, theoretical study of long

\footnotetext{
a)Electronic mail: yethiraj@chem.wisc.edu

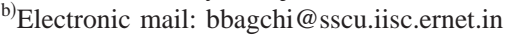

distance energy transfer between two segments in a polymer chain has been a subject of long standing interest among theoreticians. ${ }^{1,2,11,12}$ This is a nontrivial problem because of the connectivity among the monomers which makes the distance between the two reacting sites a complex, fluctuating quantity. ${ }^{11,13,14}$ Pastor, Zwanzig, and Szabo ${ }^{15}$ had earlier performed a Brownian dynamics simulation of this problem with a Heaviside sink function and compared the results with the well-known Wilemski-Fixman theory. ${ }^{16,17}$

In this work we carry out a Brownian dynamics simulation study of the energy transfer in dilute flexible polymer solutions. The polymer molecule is modeled as an ideal Gaussian chain with $N$ monomer units with segment (or Kuhn) length $b$. The donor and acceptor sites are located at opposite ends of the polymer chain. The mechanism for excitation energy transfer between the donor and acceptor sites is taken to be the Forster mechanism. ${ }^{1,10}$ In this mechanism, the singlet-singlet resonance energy transfer rate $k(R)$ is assumed to be given by

$$
k(R)=\frac{k_{F}}{1+\left(R / R_{F}\right)^{6}},
$$

where $R_{F}$ is the Forster radius, defined as the DA separation corresponding to $50 \%$ energy transfer. $k_{F}$ is the rate of excitation transfer when the separation between the donor and 
the acceptor is vanishingly small (i.e., $R / R_{F} \rightarrow 0$ ). The Forster radius is usually obtained from the overlap of the donor fluorescence with the acceptor absorption and several other available parameters. ${ }^{5}$ Note that the above form is different from the commonly used form of the Forster rate ${ }^{2}$ which is given as $k(R)=k_{F}\left(R_{F} / R\right)^{6}$. The $\left(R_{F} / R\right)^{6}$ distance dependence is not appropriate here, since it diverges at $R \rightarrow 0$, which is allowed in the Rouse chain. ${ }^{18,19}$ In a real polymer, the end-to-end distance $(R)$ never approaches zero, due to the excluded volume forces. On the other hand, one should not put the rate for distances less than the diameter of a monomer equal to zero also, as that is physically unreasonable. Thus the modified form (Eq. 1) used here seems reasonable. Our interest in the Rouse chain stems from the fact that this limit can be treated easily in theory. For example, the theory of Wilemski and Fixman ${ }^{16,17,20}$ can be readily applied to the Rouse chain because the necessary Green's function is available in analytic form.

The dynamics of Forster energy migration has been investigated traditionally via time domain measurements of the decay of the fluorescence (due to excitation transfer) from the donor. ${ }^{1,4,5,10}$ As both $k_{F}$ and $R_{F}$ are determined by the DA pair, the rate of decay of the fluorescence intensity provides a direct probe of the conformational dynamics of the polymer. Recently, this technique has been used in single molecule spectroscopy of biopolymers ${ }^{6,7}$ and proteins, ${ }^{8}$ where the distance dependence of FRET provides relevant information about the conformation and dynamics of single biopolymer. At any given time after the initial excitation, the fluorescence intensity is a measure of the "unreacted", donor concentration, that is, of the survival probability $S_{p}(t)$.

The complexity of describing the dynamics of energy transfer of polymers in solution arises from the fact that, due to chain connectivity, the Brownian motion of the monomers on the polymer are strongly correlated. The many-body nature of polymer dynamics can be described by a joint, time dependent probability distribution $P\left(\mathbf{r}^{N}, t\right)$ where $\mathbf{r}^{N}$ denotes the position of all the $N$ polymer beads at time $t$. The time dependence of the probability distribution $P\left(\mathbf{r}^{N}, t\right)$ can be described by the following reaction-diffusion equation ${ }^{16,17}$

$$
\frac{\partial}{\partial t} P\left(\mathbf{r}^{N}, t\right)=\mathcal{L}_{B}\left(\mathbf{r}^{N}, t\right) P\left(\mathbf{r}^{N}, t\right)-k(R) P\left(\mathbf{r}^{N}, t\right),
$$

where $\mathcal{L}_{B}$ is the full $3 N$ dimensional diffusion operator,

$$
\mathcal{L}_{B}(\mathbf{r}, t)=D \sum_{j=1}^{N} \frac{\partial}{\partial r_{j}} P_{\mathrm{eq}}(\mathbf{r}, t) \frac{\partial}{\partial r_{j}} \frac{P(\mathbf{r}, t)}{P_{\mathrm{eq}}(\mathbf{r}, t)},
$$

where "eq" denotes equilibrium, $R$ is the scalar distance between the two ends of the polymer chain, and $D$ is the center of mass diffusion coefficient. The solution of Eq. (2), with the sink term (last term on the right-hand side) given by the Forster expression ${ }^{1,2}$ for $k(R)$, is highly nontrivial.

In two seminal papers, Wilemski and Fixman (WF) ${ }^{16,17}$ presented a nearly analytic solution of the problem for any arbitrary sink. The WF theory has been tested, only for the average rate, by computer simulations when the sink is a Heaviside function. ${ }^{15,21} \mathrm{We}$ are not aware of any such simu- lation study with a distance dependent rate, such as Forster energy transfer. Such a study is clearly important because the end-to-end probability distribution in polymer peaks at a distance which scales as $N^{2 \nu}$. $\nu=1 / 2$ for the Rouse chain and $3 / 5$ for the self-avoiding walk $(\mathrm{SAW}){ }^{22}$ At this point it should be mentioned that Portman and Wolynes ${ }^{20}$ developed variational upper and lower bounds on the survival probability and calculated the frequency dependent survival probability for the harmonic sink and exponential sink functions. In the present study the survival probability is directly obtained in the time domain by performing BD simulations and is compared with the WF theory. We have not compared our results with the latter developments. ${ }^{11,20,23}$

The main objectives of this paper are the following. (1) To present the results of Brownian dynamics (BD) simulations of Eq. (2), with $k(R)$ given by the Forster rate [Eq. (1)]. (2) To investigate the nonexponential behavior of $S_{p}(t)$ and the viscosity dependence of the FRET rate. (3) To present a detailed comparison of the simulated rate with the WF theory. To the best of our knowledge the viscosity dependence of FRET in polymers has not been studied before. Detailed investigation into the time dependence of the survival probability shows that the $S_{p}(t)$ exhibits an interesting nonexponential behavior for the short to intermediate times, when $R_{F} \approx R_{M}$, where $R_{M}$ is the distance corresponding to the maximum in the end-to-end probability distribution of a polymer chain. The nonexponentiality is more pronounced in the case of highly viscous solutions and also for large $k_{F}$ values. Neither the observed nonexponentiality nor the fractional viscosity dependence can be explained by the WF theory. We have not made a quantitative comparison with other theoretical treatments, although such studies could be useful.

The organization of the rest of the paper is as follows. In Sec. II we describe the simulation method and in Sec. III we outline the WF theory. In Sec. IV the emergence of nonexponential behavior of $S_{p}(t)$ and the dependence of FRET rate on viscosity is discussed. In this section a comparison between the simulation results and the WF theory is also presented. We close the paper with a few conclusions in Sec. V.

\section{SIMULATION DETAILS}

Brownian Dynamics (BD) simulations are carried out for an ideal Rouse chain where the neighboring beads interact via a harmonic potential $U$ given by

$$
\beta U=\frac{3}{2 b^{2}} \sum_{j=1}^{N}\left(r_{j}-r_{j+1}\right)^{2}
$$

where $\beta^{-1}$ is the Boltzmann constant times the temperature, $r_{j}$ is the position vector of bead $j$, and the number of beads constituting the polymer chain is $N+1$. The mean square bond length is $b^{2}$, and the equilibrium root mean square end-to-end distance of the polymer chain $L$ is given by

$$
L \equiv \sqrt{\left\langle\left(r_{N+1}-r_{1}\right)^{2}\right\rangle}=\sqrt{N} b .
$$


In the Rouse model, ${ }^{18}$ since there is no excluded volume, any bead can pass through any other bead without hindrance. As a result the end-to-end distance can attain any value between zero and $N$.

In the present study a polymer chain is additionally characterized by the presence of two reactive end groups. This essentially implies that within the time interval $\Delta t$, the two end groups react with a probability $k(R) \Delta t .{ }^{24}$ The initial configuration for each trajectory is selected from Monte Carlo generated equilibrium configurations. The dynamics are then propagated using the following equation of motion:

$$
r_{j}(t+\Delta t)=r_{j}(t)+F_{j}(t) \Delta t+\Delta X^{G}(t),
$$

where $r_{j}(t)$ and $r_{j}(t+\Delta t)$ are the positions of bead $j$ at time $t$ and $t+\Delta t$, respectively. $F_{j}(t)$ is the total force acting on bead $j$ and $\Delta X^{G}(t)$ is a random Brownian displacement, taken from a Gaussian distribution with zero mean and variance $\left\langle\left(X^{G}\right)^{2}\right\rangle=2 \Delta t$. Normally distributed random numbers are generated by using the reshuffling method. ${ }^{25}$ In Eq. (6) the time and energy scales are fixed by using units where $\beta=1$, the bead diffusion coefficient $D_{0}=1$, and the mass of bead $m=1$. All the results are presented in these dimensionless units. Here a comment about the time step is required. $\Delta t$ is varied, between 0.0001 and 0.01 , depending on $R_{F}$ value. The larger the $R_{F}$, the greater the requirement for the smaller time step. For example, at $k_{F}=1$, when $R_{F}=1$ the $\Delta t=0.01$ is employed and for $R_{F}=5$, a much smaller time step, $\Delta t=0.0002$ is used.

Each trajectory generated by using the above procedure is terminated when the two end groups react. In practice this is done in the simulations as follows. At each time step, the instantaneous end-to-end distance $R$ is used in Eq. (1) to calculate the distance dependent rate constant $k(R)$. The trajectory is then terminated with a probability $k(R)$, i.e., a uniformly distributed random number between 0 and 1 is selected and if this random number is less than $k(R) \Delta t$, then the trajectory is terminated; otherwise, the trajectory is continued. Averages are obtained over many such trajectories. This procedure generates an irreversible FRET. ${ }^{24}$

Each polymer chain is equilibrated for $10^{5}$ time steps before the reaction is switched on. Subsequently, 50000 to 100000 trajectories with different initial configurations are generated and the survival probability $S_{p}(t)$ is obtained by averaging over all the trajectories. This procedure is systematically applied for the polymer chains with $N=20,50$, and 100. As a check of the simulation method, the results of Pastor, Zwanzig, and Szabo (PZS) ${ }^{15}$ on the mean first passage time, with Heaviside sink function of infinite strength, are reproduced. Our simulation results agreed with those of $\mathrm{PZS}$, within the uncertainty given by PZS.

\section{WILEMSKI-FIXMAN THEORY}

Wilemski and Fixman (WF) ${ }^{16,17}$ developed an elegant theory for the diffusion limited intrachain reaction of a flexible polymer. Recently, Portman and Wolynes ${ }^{20}$ presented a simple and straightforward derivation of the WF scheme. In WF theory, to account for the chemical reaction, WF added a sink term $\mathcal{S}$ to the many-body diffusion equation, just as in Eq. (2). The WF equation of motion is given by

$$
\frac{\partial}{\partial t} P\left(\mathbf{r}^{N}, t\right)+\mathcal{L}_{B} P\left(\mathbf{r}^{N}, t\right)=-k_{0} \mathcal{S}(R) P\left(\mathbf{r}^{N}, t\right) .
$$

In the notation of the present work,

$$
k_{0}=k_{F}
$$

and

$$
\mathcal{S}(R)=\frac{1}{1+\left(R / R_{F}\right)^{6}},
$$

where the operator $\mathcal{L}_{B}\left(\mathbf{r}^{N}, t\right)$ is given by Eq. (3). As mentioned in the Introduction, the treatment of WF is general and can be applied to a reaction with arbitrary sink.

The survival probability $S_{p}(t)$ is defined as the probability that the chain has not reacted after time $t$ and is given by

$$
S_{p}(t)=\int P\left(\mathbf{r}^{N}, t\right) d \mathbf{r}_{1} d \mathbf{r}_{2} \cdots d \mathbf{r}_{N}
$$

In order to obtain the survival probability, WF made a closure approximation, according to which the Laplace transform of $S_{p}(t)$ is approximated as

$$
\hat{S}_{p}(s)=\frac{1}{s}-\frac{k v_{\mathrm{eq}}}{s^{2}\left(1+k \hat{D}(s) / v_{\mathrm{eq}}\right)},
$$

where $k$ is the momentum transfer variable and $s$ is the Laplace transform variable. It is important to note that the above equation is a zeroth order approximation which, as discussed by WF, limits the validity of the theory to the case when the deviation from equilibrium distribution at any time is small. The final form of WF theory is expressed in terms of a frequency dependent function $D(s)$ which is given by

$$
\hat{D}(s)=\int_{0}^{\infty} e^{-s t} D(t) d t,
$$

which is the Laplace transform of sink-sink time correlation function $D(t) . D(t)$ is given by

$$
D(t)=\int d^{3} \mathbf{R}_{1} \int d^{3} \mathbf{R}_{2} \mathcal{S}\left(\mathbf{R}_{1}\right) \mathcal{S}\left(\mathbf{R}_{2}\right) G\left(\mathbf{R}_{1}, \mathbf{R}_{2}, t\right) P_{\mathrm{eq}}\left(\mathbf{R}_{2}\right),
$$

where the equilibrium end-to-end distribution function $P_{\text {eq }}(\mathbf{R})$ is given by

$$
P_{\text {eq }}(\mathbf{R})=\left(\frac{3}{2 \pi L^{2}}\right)^{3 / 2} \exp \left(\frac{-3 R^{2}}{2 L^{2}}\right) .
$$

The Green's function $G$ appearing in Eq. (13) is given by

$$
\begin{aligned}
G\left(\mathbf{R}_{1}, \mathbf{R}_{2}, t\right)= & \left(\frac{3}{2 \pi L^{2}}\right)^{3 / 2}\left(\frac{1}{\left[1-\rho^{2}(t)\right]^{3 / 2}}\right) \\
& \times \exp \left(-\frac{R_{1}^{2}-2 \rho(t) \mathbf{R}_{1} \cdot \mathbf{R}_{2}+R_{2}^{2} \rho^{2}(t)}{2 L^{2}\left[1-\rho^{2}(t)\right]}\right),
\end{aligned}
$$

where $\rho(t)$ is the normalized time correlation function of end-to-end vector defined as $\langle\mathbf{R}(0) \cdot \mathbf{R}(t)\rangle /\left\langle R^{2}\right\rangle$, which can be obtained analytically and is given by the following equation, 


$$
\rho(t)=\frac{8}{\pi^{2}} \sum_{l ; \text { odd }} \frac{4}{l^{2}} \exp \left(-\lambda_{l} t\right)
$$

Note that in the above equation, summation includes only the odd values of $l$. If we neglect the excluded volume and the hydrodynamic interactions (to account for the Rouse model), $\lambda_{l}$ is given by ${ }^{16,17}$

$$
\lambda_{l}=3 D_{0}(l \pi / N b)^{2} .
$$

Finally $v_{\text {eq }}$ is defined as

$$
\lim _{t \rightarrow \infty} D(t)=\left(v_{\text {eq }}\right)^{2} \text {. }
$$

Note that $v_{\text {eq }}$ is the rate when the distribution of the polymer ends is at equilibrium. Thus $v_{\text {eq }}$ gives the initial rate of decay of $S_{P}(t)$ and can be estimated from the transient behavior. In most cases the rate of decay should become progressively smaller, as the population from the sink region decreases, as the reaction proceeds.

By using the above set of equations and after averaging over all the angles, one can write the sink-sink time correlation function $[D(t)]$ in the following form, ${ }^{15}$

$$
\begin{aligned}
D(t)= & \left(\frac{3}{2 \pi L^{2}}\right)^{3} \frac{1}{\left[1-\rho^{2}(t)\right]^{3 / 2}} \\
& \times \int_{0}^{\infty} 4 \pi R_{1}^{2} \mathcal{S}\left(R_{1}\right) d R_{1} \int_{0}^{\infty} 4 \pi R_{2}^{2} \mathcal{S}\left(R_{2}\right) d R_{2} \\
& \times \exp \left(-\frac{3\left(R_{1}^{2}+R_{2}^{2}\right)}{2 L^{2}\left[1-\rho^{2}(t)\right]}\right) \\
& \times \frac{\sinh \left\{\left[3 \rho(t) R_{1} R_{2}\right] /\left(L^{2}\left[1-\rho^{2}(t)\right]\right)\right\}}{\left[3 \rho(t) R_{1} R_{2}\right] /\left(L^{2}\left[1-\rho^{2}(t)\right]\right)} .
\end{aligned}
$$

Once the choice of the sink function is specified, it is straightforward to calculate the survival probability by utilizing the above set of equations. WF's choice was the Heaviside sink function. Later Doi $^{26}$ showed that the WF method is easy to apply if the Heaviside sink function is replaced with a Gaussian sink function. Battezzatti and Perico ${ }^{27}$ studied the dependence of the rate on the choice of sink function within the frame work of WF theory and supported the WF closure approximation. In this study $S_{p}(t)$ is obtained from the Laplace inversion of Eq. (11). In doing so we use the Stehfest algorithm. ${ }^{28}$ In the notation of present work, the final form of the sink-sink time correlation function can be written as

$$
\begin{aligned}
D(t)= & \left(\frac{3}{2 \pi L^{2}}\right)^{3} \frac{1}{\left(1-\rho^{2}\right)^{3 / 2}} \\
& \times \int_{0}^{\infty} 4 \pi R_{1}^{2}\left(\frac{R_{F}^{6}}{R_{1}^{6}+R_{F}^{6}}\right) d R_{1} \int_{0}^{\infty} 4 \pi R_{2}^{2}\left(\frac{R_{F}^{6}}{R_{2}^{6}+R_{F}^{6}}\right) d R_{2} \\
& \times \exp \left(-\frac{3\left(R_{1}^{2}+R_{2}^{2}\right)}{2 L^{2}\left[1-\rho^{2}(t)\right]}\right) \\
& \times \frac{\sinh \left\{\left[3 \rho(t) R_{1} R_{2}\right] /\left(L^{2}\left[1-\rho^{2}(t)\right]\right)\right\}}{\left[3 \rho(t) R_{1} R_{2}\right] /\left(L^{2}\left[1-\rho^{2}(t)\right]\right)}
\end{aligned}
$$

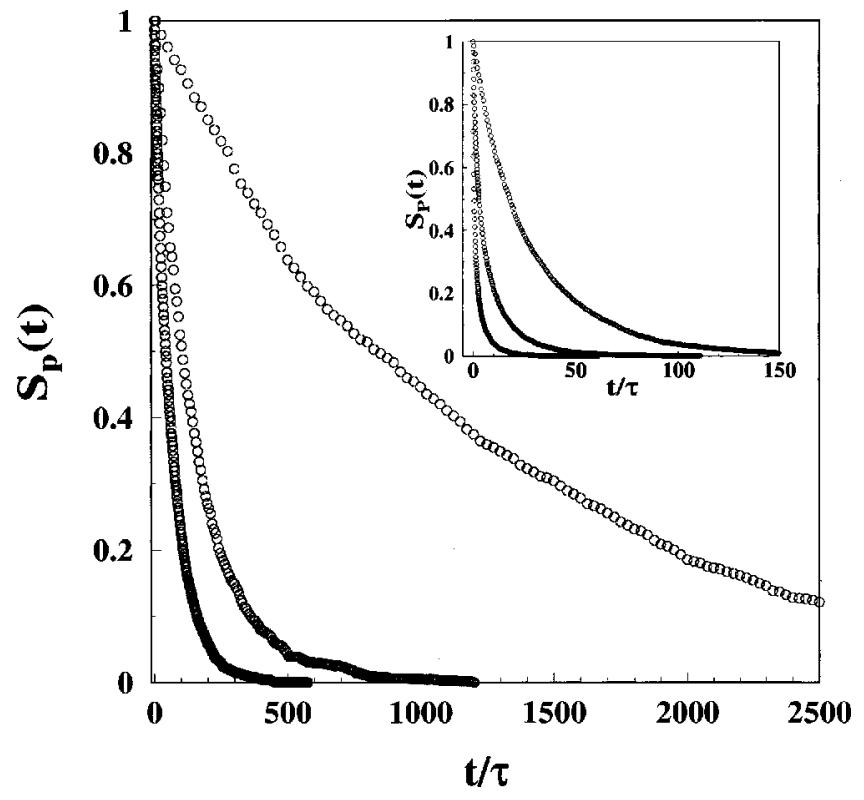

FIG. 1. Brownian dynamics (BD) simulation results for the survival probability $S_{p}(t)$ are plotted as a function of scaled time for $N=50$ for several values of $k_{F}$. The main figure shows the $S_{p}(t)$ for $R_{F}=1$ and the inset shows that for $R_{F}=5$. In both the figures, curves from top to bottom correspond to $k_{F}=0.1,1$, and 10 , respectively.

\section{RESULTS AND DISCUSSION}

\section{A. Survival probability}

Figure 1 depicts the time dependence of the survival probability $S_{p}(t)$ for $N=50$. The main figure shows the $S_{p}(t)$ for $R_{F}=1$, while the inset shows the result for $R_{F}$ $=5$. In both figures $k_{F}$ is varied from 0.1 to 10 , that is, two orders of magnitude. This figure demonstrates the strong dependence of the decay of $S_{P}(t)$ on $R_{F}$. In both the figures, curves from top to bottom represent the $S_{p}(t)$ for $k_{F}=0.1,1$, and 10 , respectively. The maximum survival time for $R_{F}$ $=1$ is more than an order of magnitude larger than that of $R_{F}=5$, at a fixed $k_{F}$ value. This strong dependence of decay of $S_{p}(t)$ on $R_{F}$ could be potentially useful in unravelling the mechanism and the dynamics of energy transfer. Note that the earlier experiments ${ }^{3,5}$ (which fit the quantum yield to the Forster expression) obtained values which were rather large, even larger than the root mean square radius of the polymer chain. This could have been due to the use of an equilibrium end-to-end probability distribution in the fitting, instead of a time dependent distribution. In model calculations, one usually assumes a small value of $R_{F}$ (often in the form of a Heaviside sink function).

It is not difficult to understand the above results qualitatively. For an ideal Gaussian chain, the maximum in the probability $\left[4 \pi R^{2} P(R)\right]$, that the two ends are separated by a distance $R$, is located at $R_{M}[=\sqrt{(2 N / 3)} b]$. For $N=50$, $R_{M} \approx 5.773 \mathrm{~b}$. Therefore when $R_{F}=5$, the decay is facilitated by the presence of a large fraction of the population at a separation where the transfer rate is large. This can explain the relatively faster decay for $R_{F}=5$ (inset). However, the situation is completely different for $R_{F}=1$. Here the probability of finding a polymer with this small end-to-end distance is negligible and the transfer rate where the bulk of the 


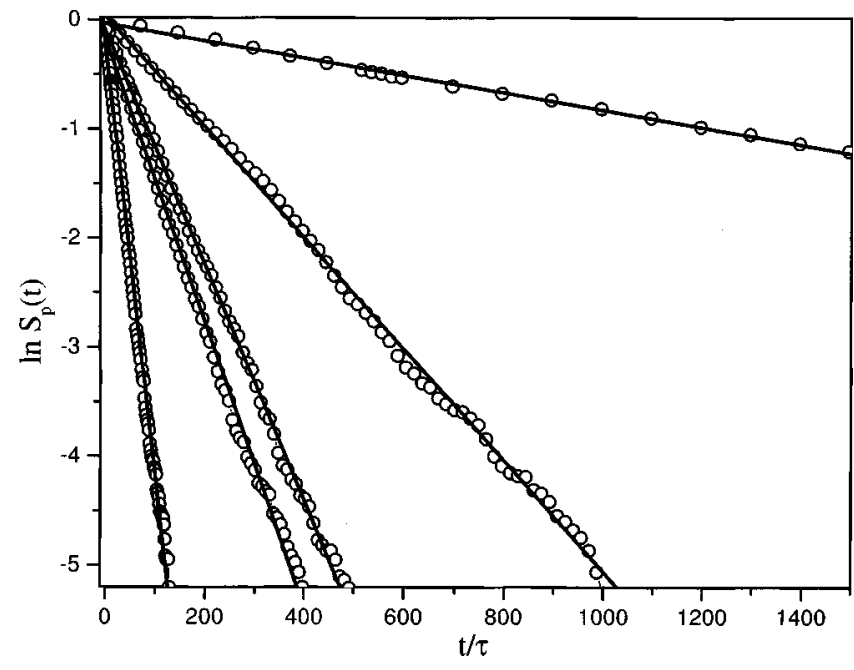

FIG. 2. The semilog plot of $S_{p}(t)$ against the reduced time for $N=50$ at $R_{F}=1$. Symbols show the simulation result, while the straight lines are linear fits to the long time, indicating the eventual exponential behavior. Curves from top to bottom show the result for $\widetilde{k}_{F}=0.1,1,5,10$, and 50 .

population is located is very small because of the strong $R$ dependence of the Forster energy transfer rate. Therefore the decay of the survival probability is slow for short times and is determined by the interplay between the diffusion and the rate. This explains the decay nature of $S_{p}(t)$ curves, shown in Fig. 1.

The above discussion also suggests that the decay of the survival probability can depend strongly on the length of the polymer chain. This is because the Forster radius for a given DA pair is likely to be independent of the length of the polymer chain. However, this dependence is not trivial, we hope to address it elsewhere.

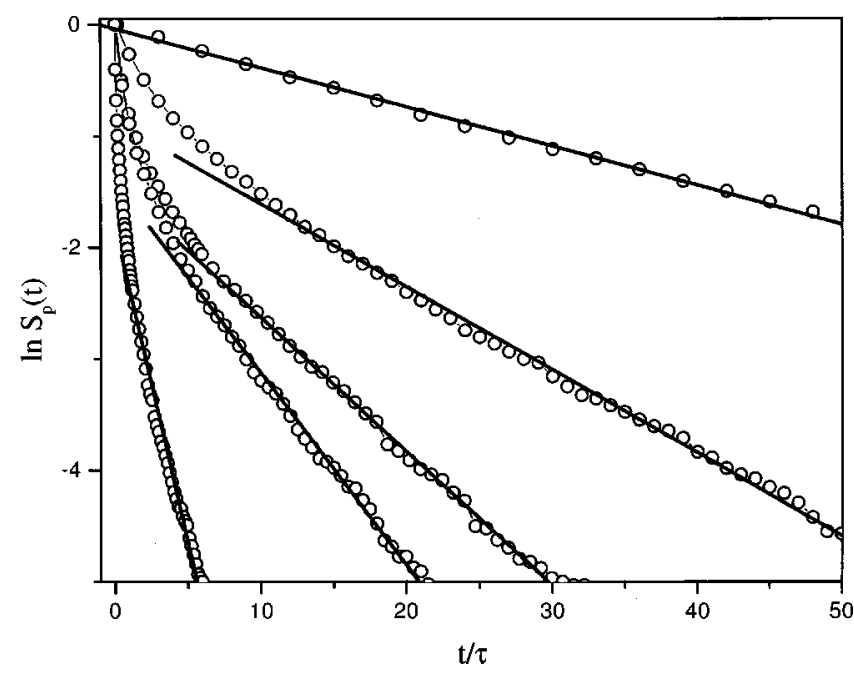

FIG. 3. Same as Fig. 2 but for $R_{F}=5$. This figure clearly shows that the crossover time of $S_{p}(t)$ from the nonexponential behavior at short to intermediate times to the exponential decay at longer times increases with increasing the $\widetilde{k}_{F}$ at constant $k_{F}$.

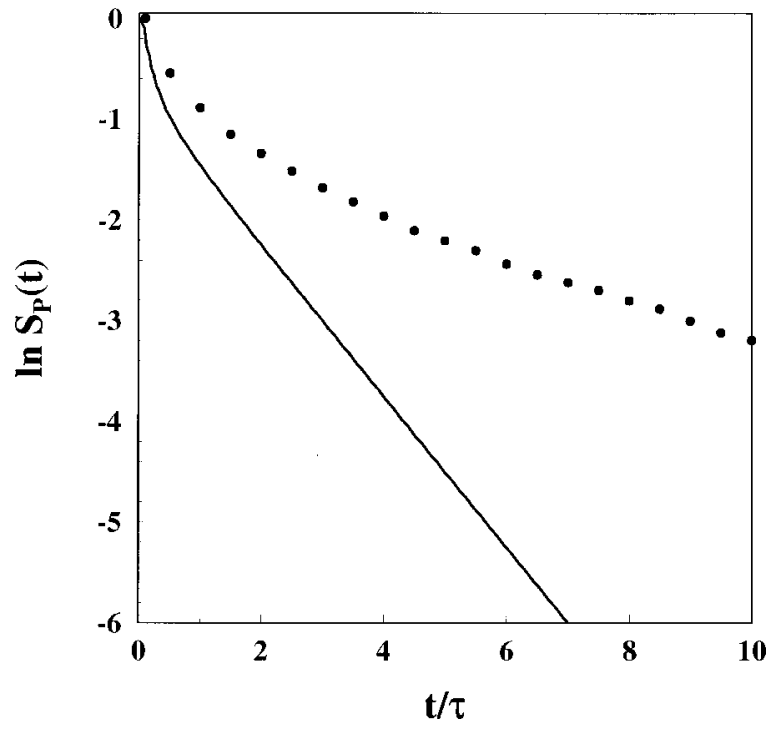

FIG. 4. The semilog plot of $S_{p}(t)$ obtained from simulation (symbols) and WF theory (line) is shown, as a function of time, at $R_{F}=5$ and $\widetilde{k}_{F}=10$ for $N=50$. In this limit, simulated $S_{p}(t)$ shows highly nonexponential behavior for a relatively longer time, compared to that obtained from WF theory.

\section{B. Nonexponentiality of $S_{p}(t)$ and the fractional viscosity dependence of the rate}

In this section we explore the connection between the observed nonexponentiality of $S_{p}(t)$ and a possible fractional viscosity dependence of the average rate. For the sake of generality, the results are presented here in terms of a dimensionless quantity $\widetilde{k}_{F}$, defined as $k_{F} / D$. Thus the results presented here can be interpreted in two ways, $\widetilde{k}_{F}$ can be varied by keeping the center of mass diffusion $(D)$ constant while varying $k_{F}$. Another way is to keep $k_{F}$ constant but vary $D$. In the latter case, variation in $D$ affects the viscosity $(\eta)$ in an exactly opposite manner. Thus at constant $k_{F}$, large values

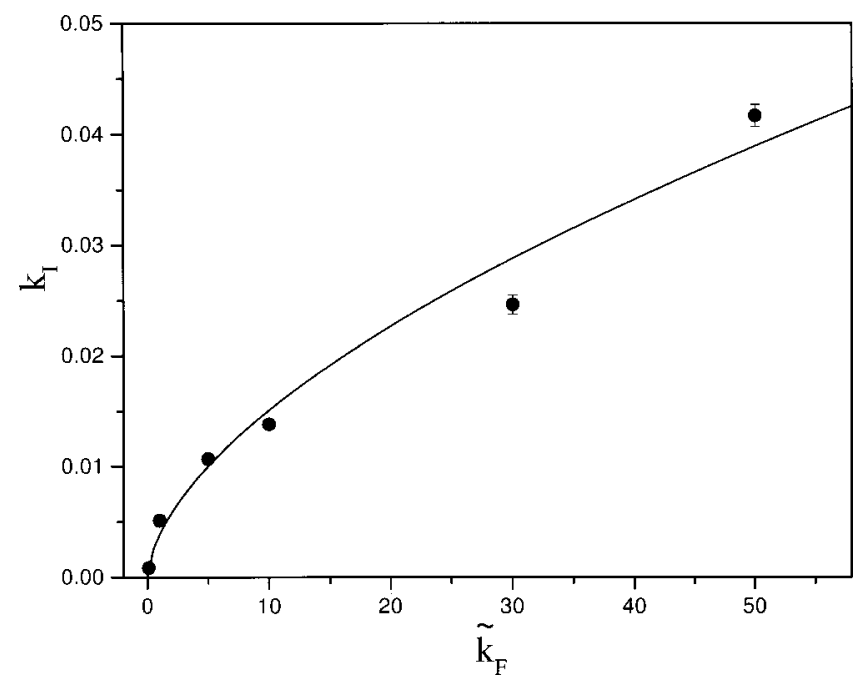

FIG. 5. The average FRET rate $\left(k_{I}\right)$ obtained from BD simulations is plotted against $\widetilde{k}_{F}$ for $N=50$ at $R_{F}=1$. Symbols show the simulation result, while the full line is the fit to Eq. (21). Error bars represent the uncertainties and are within $10 \%$. The exponent $\alpha$ [in Eq. (21)] is calculated as -0.58 , indicating the fractional viscosity dependence of $k_{I}$. 


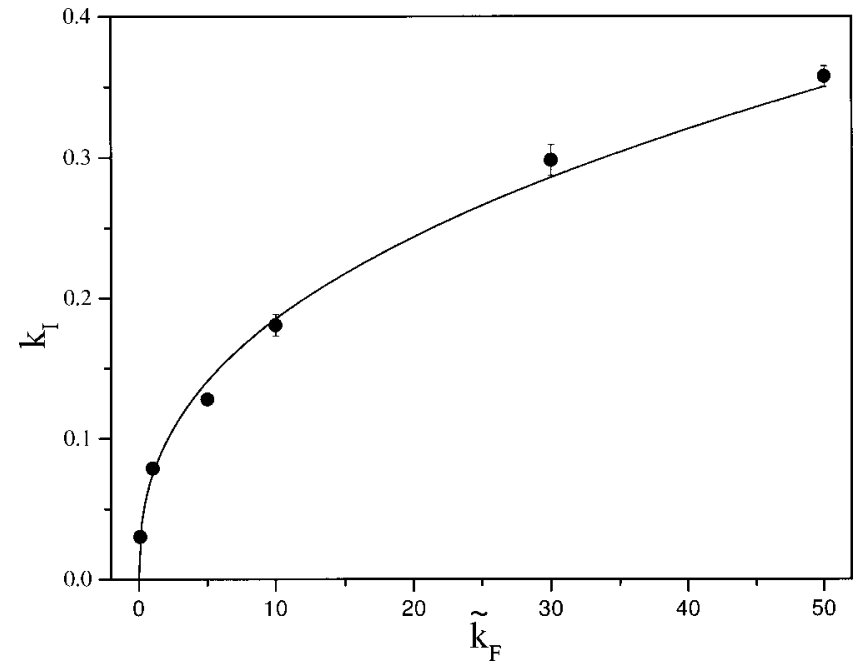

FIG. 6. Same as Fig. 5 but for $R_{F}=5$. The exponent $\alpha$ [in Eq. (21)] is calculated as 0.395 , indicating the fractional viscosity dependence of $k_{I}$.

of $\widetilde{k}_{F}$ represent solutions of high viscosity and small $\widetilde{k}_{F}$ values represent solutions of low viscosity. Figures 2 and 3 show the semilog plot of survival probability for varying values of $\widetilde{k}_{F}$ at $R_{F}=1$ and $R_{F}=5$, respectively. According to the above discussion, these figures show the effect of viscosity on $S_{p}(t)$ at a fixed $k_{F}$ value. In both the figures, simulation results are represented by symbols, while the straight lines are linear fits showing the extent of exponential behavior. At lower $R_{F}$ values the decay of survival probability retains the exponential behavior over the entire range of viscosity probed in this study (Fig. 2). On the other hand, when $R_{F}$ is comparable to $R_{M}$, the crossover time of $S_{p}(t)$ from the nonexponential behavior at short to intermediate times to the exponential behavior at long times increases, as the viscosity of the polymer solution increases (see Fig. 3). That is, the extent of nonexponentiality increases with solution viscosity. The large exponentiality observed in simulations for $R_{F} \approx R_{M}$ and large $k_{F}$ cannot be explained by the WF theory, as shown in Fig. 4. (Details of the comparison of WF theory with simulation results are discussed later).

Note that the viscosity dependence of FRET could act as an important marker of polymer/protein folding. In order to analyze the viscosity dependence of FRET rate $\left(k_{I}\right)$ in more detail, we have adopted the following well-known form,

$$
k_{I}=A \eta^{-\alpha},
$$

$A$ is a characteristic constant of the reaction. The average FRET rate is defined as

$$
k_{I}^{-1}=\int_{0}^{\infty} S_{p}(t) d t
$$

Figures 5 and 6 show the variation of $k_{I}$ against $\widetilde{k}_{F}$, for $N$ $=50$, at $R_{F}=1$ and $R_{F}=5$, respectively. The FRET rate $\left(k_{I}\right)$ obtained from BD simulations is shown by symbols, while the full lines are the fits to Eq. (21), in both the figures. For $R_{F}=1$ the exponent $\alpha$ is equal to 0.58 (Fig. 5) and for $R_{F}$ $=5, \alpha$ is found to have a value of 0.395 (Fig. 6). These two figures clearly suggest that the FRET rate can exhibit a frac-
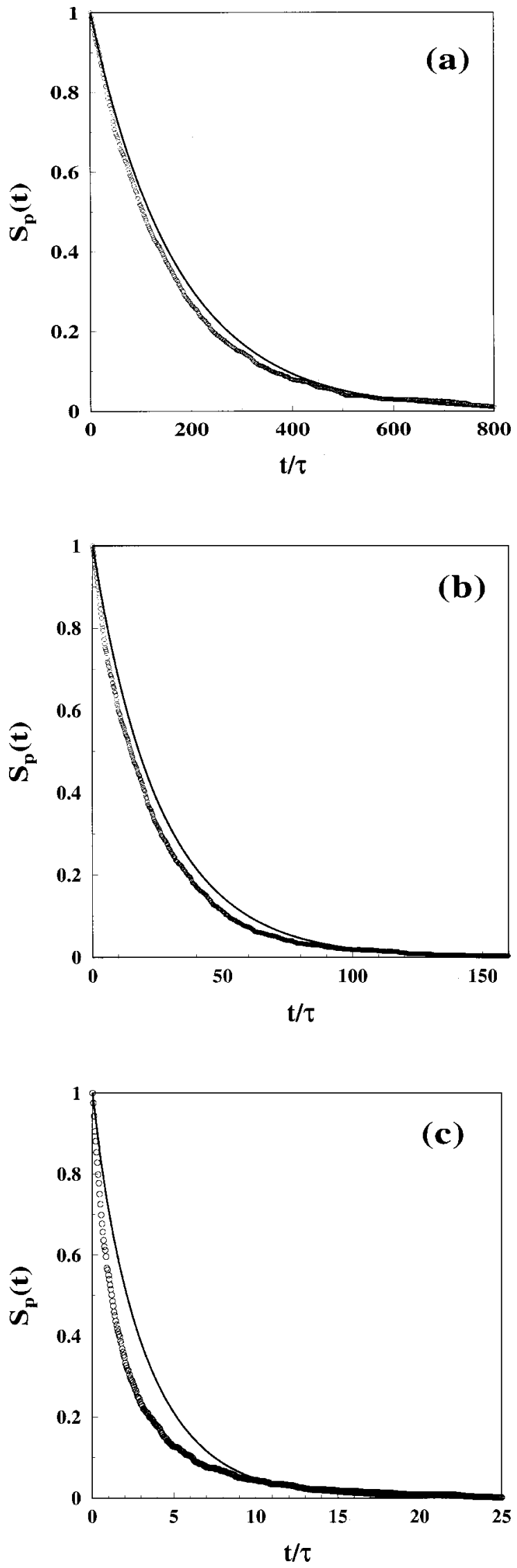

FIG. 7. BD simulation results (symbols) for $S_{p}(t)$ compared with the predictions of the WF theory (lines) for $N=50$, for various values of $R_{F}$, at $k_{F}=1$. Figures (a)-(c) show the comparison for $R_{F}=1,2$, and 5, respectively. Theory and simulation agrees well in the limit of small $R_{F}[(\mathrm{a})]$. Agreement becomes progressively poorer as $R_{F}$ is increased [(b) and (c)]. 


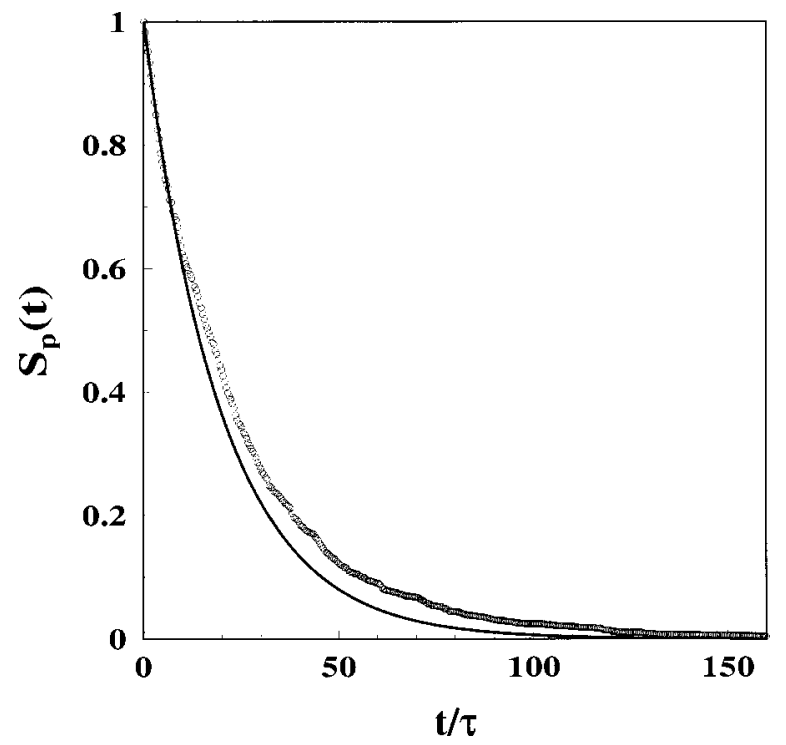

FIG. 8. Comparison of simulation results (symbols) for $S_{p}(t)$ to predictions of the WF theory (line) for a large Forster rate, namely $k_{F}=10$ at $R_{F}=1$ for $N=50$. The agreement is satisfactory only at short times.

tional viscosity dependence of the form $\eta^{-\alpha}$, where the exponent $\alpha$ can attain a value much less than unity. This fractional viscosity dependence occurs due to a competition between reaction and diffusion and is well-known in the reaction dynamics literature. ${ }^{29,30}$ Another possible reason is the collective many-body nature of the dynamics. This is reflected in the non-Markovian equation of motion for the reduced equation of motion of $P(R, t)$. We found that the WF theory fails to capture the fractional viscosity dependence. For example, it gives a value of $\alpha=0.91$ for $R_{F}=1$, when the simulated value is 0.59 .

\section{Comparison with WF theory}

Before discussing the results we describe the scaling used to compare the simulation results to theoretical predictions. In this work, time is measured in units of $b^{2} / D_{0}$ and the rate constant is measured in units of $D_{0} / b^{2}$. In the original WF theory, time was measured in units of $6 D / L^{2}$, where $D$ is the center of mass diffusion constant and $L^{2}$ is the mean square end-to-end distance. The units of the rate constant used here differ from that of WF by a factor of $6 / N^{2}$ (note that $D=D_{0} / N$ and $L^{2}=N b^{2}$ in the free draining limit). The Forster radius is scaled by the bead diameter $b$. Another important parameter in this problem is the root mean square end-to-end distance of the polymer, as this determines the end-to-end distribution. Although we have carried out simulations for $N=20,50$, and 100 , in this report we shall concentrate mostly on $N=50$.

In Figs. 7(a), 7(b), and 7(c), the survival probabilities obtained from simulation are compared with the WF theory prediction for various values of $R_{F}$, at $k_{F}=1$. In all the figures symbols show the simulation results while the full line represents the predictions of WF theory. WF theory predictions are in good agreement with that of the simulations at both short and long times for $R_{F}=1$ [Fig. 7(a)]. As the Forster radius is increased, the agreement becomes progressively

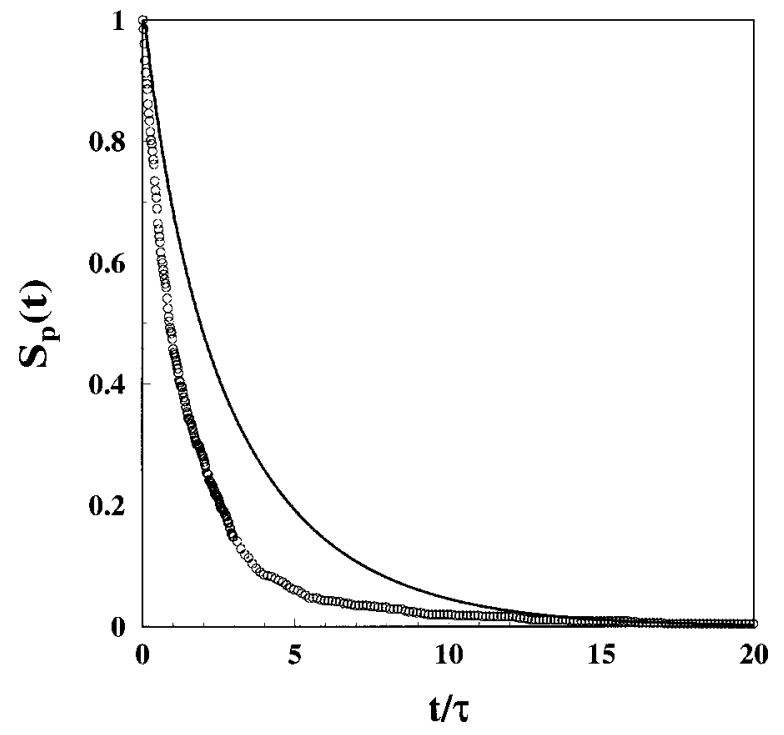

FIG. 9. Comparison of simulation results (symbols) for $S_{p}(t)$ to predictions of the WF theory (line) for a longer chain $(N=100)$ at $R_{F}=8$ and $k_{F}=1$.

poorer [Fig. 7(b)]. For the $R_{F}$ values comparable to $R_{M}$, the agreement between WF theory and simulation results is not satisfactory [shown in Fig. 7(c)]. We have also compared the WF theory predictions with the simulation results at larger Forster rate. This is shown in Fig. 8, where the comparison of $S_{P}(t)$ is shown at $k_{F}=10$ for $R_{F}=1$ and $N=50$. Here again the agreement is satisfactory only at short times. In Fig. 9 we show the comparison between the simulation results and the WF theory for $N=100$ at $R_{F}=8$ and $k_{F}=1$. We choose $R_{F}=8$ because for $N=100, R_{M} \approx 8.16$. In this case the comparison is qualitatively similar to the corresponding case for $N=50$, the WF theory breaks down at intermediate times. Note that in many applications of FRET, small sized polymers are involved, as in proteins ${ }^{8}$ and oligomers. ${ }^{3}$ The above results could be useful to such cases.

Our results are consistent with the conclusion of the previous studies ${ }^{15,26}$ that considered only the average rate by using the Heaviside sink and other sink functions. These studies found that the WF theory works better for a sink with a smaller reaction radius than for one with a larger radius. The present study suggests that in the case of the Forster sink, the WF theory works well as the Forster radius decreases. WF used an approximate expression for $\rho(t)$ and so we have checked the accuracy of their approximation for $\rho(t)$ also. Figure 10 compares the simulated end-to-end vector time correlation function $[\rho(t)]$, for $N=50$, with the approximate expression used by WF [Eq. (16)]. The agreement is good and improves further for larger $N$. Thus the use of the approximate $\rho(t)$ is not the reason for the failure of WF theory. The inability of the Wilemski-Fixman theory to explain the time dependence of the survival probability at $R_{F}$ $\approx R_{M}$ values is, however, not very surprising. This is because WF made a local equilibrium assumption, which essentially implies that this theory is efficient only when the system is not too far from the equilibrium. This assumption works well for the smaller reaction rates but leads to the erroneous result for the larger reaction rates and also where population is small. When $R_{F} \approx R_{M}$, FRET rate is facilitated 


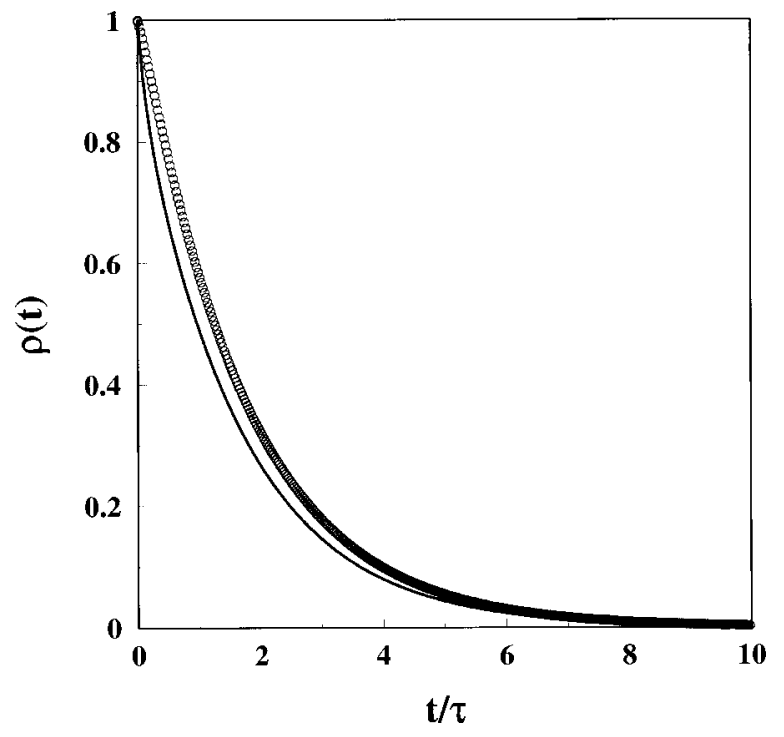

FIG. 10. The end-to-end vector time correlation function $\rho(t)$ plotted against the scaled time for a polymer of mean square end-to-end distance, $L^{2}=50 b^{2}$. Symbols represent the simulated $\rho(t)$ and the full line shows the $\rho(t)$ obtained by using the approximate expression of WF [Eq. (16)].

by the presence of a large fraction of population with the required end-to-end separation. This essentially drives the system away from the equilibrium. Clearly, in this situation, the local equilibrium assumption is not valid.

\section{CONCLUSIONS}

Let us first summarize the main results of this paper. Detailed Brownian dynamics simulations of fluorescence resonance energy transfer (FRET) between the two ends of an ideal Gaussian chain have been carried out. As noted by previous workers, ${ }^{15}$ this apparently simple problem is actually highly nontrivial because even a single polymer molecule is a many body problem. We have calculated survival probability for a large number of values of the transfer rate $k_{F}$ and the Forster radius $R_{F}$. The survival probability is exponential-like for the smaller values of $R_{F}$ but shows highly nonexponential behavior in the short to intermediate times with increasing $R_{F}$. The same is true for the Forster rate $k_{F}$.

It is found that the viscosity of solution can affect the survival probability and thus the FRET rate to a great extent. The nonexponential behavior of $S_{p}(t)$ is more prominent in the case of highly viscous solutions. For smaller $R_{F}$ values the effect of viscosity on the decay profile of $S_{p}(t)$ is negligible. We have predicted that the FRET rate can exhibit a fractional viscosity dependence $\left(\sim \eta^{-\alpha}\right)$, where the exponent $\alpha$ can attain a value as low as 0.39 depending on the Forster radius and $\tilde{k}_{F}$.

We compare the results of the simulation with the wellknown theory of Wilemski and Fixman at the level of $S_{p}(t)$ and find that the theory is reliable when the Forster radius $R_{F}$ is small compared to $R_{M}$ and the transfer rate $k_{F}$ is comparable to or smaller than the monomer diffusion rate $D_{0} / b^{2}$. However, the agreement is not satisfactory in the limit, when $R_{F}$ is either comparable or equal to $R_{M}$ and $k_{F}$ is large.
Neither the observed nonexponentiality nor the fractional viscosity dependence can be explained by the WF theory. The present studies suggest that for many realistic situations we need to go beyond the zeroth order approximation employed in the WF theory. In this regard, the work of Portman and Wolynes ${ }^{20}$ may prove useful.

The techniques employed in this work could be employed in other related fields. The distance dependent rate appears in several other chemical processes, where the rate of transfer is known to show an exponential distance dependence. One such example is the electron transfer reactions; it is of interest to use the method employed here to that problem as well. Another important long standing problem is the study of reactions in realistic polymer chains with excluded volume and hydrodynamic interactions. Finally, the simulation results obtained here should be analyzed by using the theory of Portman and Wolynes. ${ }^{20}$ Work in these directions is in progress.

\section{ACKNOWLEDGMENTS}

This work was supported in part by the Council of Scientific and Industrial Research (CSIR) and the Department of Science and Technology (DST), India. G. Srinivas thanks CSIR, New Delhi, India for a research fellowship. A part of this work was carried out when A. Yethiraj was on sabbatical leave at the Indian Institute of Science, Bangalore, and the Jawaharlal Nehru Center for Advanced Scientific Research, Jakkur, Bangalore. A.Y. gratefully acknowledges financial support from the Jawaharlal Nehru Center for Advanced Scientific Research, Bangalore, India, the Alfred P. Sloan Foundation, and the National Science Foundation (through Grants No. CHE 9502320 and No. CHE 9732604).

${ }^{1}$ Th. Forster, Ann. Phys. (Leipzig) 2, 55 (1948).

${ }^{2}$ Th. Forster, in Modern Quantum Chemistry, Istanbul Lectures, Part III: Action of Light and Organic Crystals, edited by O. Sinanoglu (Academic, New York, 1965).

${ }^{3}$ L. Stryer and R. P. Haugland, Proc. Natl. Acad. Sci. U.S.A. 58, 719 (1967).

${ }^{4}$ G. B. Birks, in Photophysics of Aromatic Molecules (Wiley-Interscience, London, 1970), p. 576.

${ }^{5}$ E. Haas, M. Wilchek, E. Katchalski-Katzir, and I. Z. Steinberg, Proc. Natl. Acad. Sci. U.S.A. 72, 1807 (1975).

${ }^{6}$ T. Ha, A. Y. Ting, J. Liang, W. B. Caldwell, A. A. Deniz, D. S. Chemla, P. G. Schultz, and S. Weiss, Proc. Natl. Acad. Sci. U.S.A. 96, 893 (1999).

${ }^{7}$ A. A. Deniz, M. Dahan, J. P. Grunwell, T. Ha, A. E. Faulhaber, D. S. Chemla, S. Weiss, and P. G. Schultz, Proc. Natl. Acad. Sci. U.S.A. 96, 3670 (1999).

${ }^{8}$ A. A. Deniz, T. A. Laurence, G. S. Beligere, M. Dahan, A. B. Martin, D. S. Chemla, P. E. Dawson, P. G. Schultz, and S. Weiss, Proc. Natl. Acad. Sci. U.S.A. 97, 5179 (2000).

${ }^{9}$ A. Grinvald, E. Haas, and I. Z. Steinberg, Proc. Natl. Acad. Sci. U.S.A. 69, 2273 (1972)

${ }^{10}$ C. R. Cantor and P. Pechukas, Proc. Natl. Acad. Sci. U.S.A. 68, 2099 (1971)

${ }^{11}$ J. Wang and P. G. Wolynes, J. Chem. Phys. 110, 4812 (1999).

${ }^{12}$ M. Doi, Chem. Phys. 11, 107 (1975); S. Sunagawa and M. Doi, Polym. J. (Singapore) 7, 604 (1975); 8, 239 (1976).

${ }^{13}$ H. Frauenfelder, S. Sligar, and P. G. Wolynes, Science 254, 1598 (1991).

${ }^{14}$ Y. B. Zeldovich, A. A. Ruzmaikin, and D. D. Sokoloff, The Almighty Chance (World Scientific, Singapore, 1990).

${ }^{15}$ R. W. Pastor, R. Zwanzig, and A. Szabo, J. Chem. Phys. 105, 3878 (1996).

${ }^{16}$ G. Wilemski and M. Fixman, J. Chem. Phys. 60, 866 (1974).

${ }^{17}$ G. Wilemski and M. Fixman, J. Chem. Phys. 60, 878 (1974). 
${ }^{18}$ P. E. Rouse, J. Chem. Phys. 21, 1272 (1953).

${ }^{19}$ M. Doi and S. F. Edwards, The Theory of Polymer Dynamics (Oxford University Press, Oxford, 1986).

${ }^{20}$ J. J. Portman and P. G. Wolynes, J. Phys. Chem. A 103, 10602 (1999).

${ }^{21}$ R. Zwanzig, Acc. Chem. Res. 23, 148 (1990), and reference therein.

${ }^{22}$ P. de Gennes, Scaling Concepts in Polymer Physics (Cornell University Press, Ithaca, 1979).

${ }^{23}$ J. Wang and P. G. Wolynes, Phys. Rev. Lett. 74, 4317 (1994).

${ }^{24}$ G. Srinivas and B. Bagchi, Chem. Phys. Lett. 328, 420 (2000).
${ }^{25}$ W. H. Press, S. A. Teulolsky, W. T. Vellerling, and B. P. Flannery, $N u$ merical Recipes in Fortran (Cambridge University Press, Cambridge, England, 1986).

${ }^{26}$ M. Doi, Chem. Phys. 9, 455 (1975).

${ }^{27}$ M. Battezzatti and A. Perico, J. Chem. Phys. 74, 4527 (1981)

${ }^{28}$ H. Stehfest, Commun. ACM 13, 624 (1970).

${ }^{29}$ B. Bagchi and G. R. Fleming, J. Phys. Chem. 78, 7375 (1983).

${ }^{30}$ G. R. Fleming, Chemical Applications of Ultrafast Spectroscopy (Oxford, New York, 1986), Chap. 6. 